\title{
Split Hopkinson Resonant Bar Test for Sonic-Frequency Acoustic Velocity and Attenuation Measurements of Small, Isotropic \\ Geological Samples
}

\author{
Seiji Nakagawa
}

Earth Sciences Division, Lawrence Berkeley National Laboratory, 1 Cyclotron Road, Berkeley, California, 94720. 


\begin{abstract}
Mechanical properties (seismic velocities and attenuation) of geological materials are often frequency dependent, which necessitates measurements of the properties at frequencies relevant to a problem at hand. Conventional acoustic resonant bar tests allow measuring seismic properties of rocks and sediments at sonic frequencies (several kilohertz) that are close to the frequencies employed for geophysical exploration of oil and gas resources. However, the tests require a long, slender sample, which is often difficult to obtain from the deep subsurface or from weak and fractured geological formations. In this paper, an alternative measurement technique to conventional resonant bar tests is presented. This technique uses only a small, jacketed rock or sediment core sample mediating a pair of long, metal extension bars with attached seismic source and receiver-the same geometry as the split Hopkinson pressure bar test for large-strain, dynamic impact experiments. Because of the length and mass added to the sample, the resonance frequency of the entire system can be lowered significantly, compared to the sample alone. The experiment can be conducted under elevated confining pressures up to tens of $\mathrm{MPa}$ and temperatures above $100^{\circ} \mathrm{C}$, and concurrently with x-ray $\mathrm{CT}$ imaging. The described Split Hopkinson Resonant Bar (SHRB) test is applied in two steps. First, extension and torsion-mode resonance frequencies and attenuation of the entire system are measured. Next, numerical inversions for the complex Young's and shear moduli of the sample are performed. One particularly important step is the correction of the inverted Young's moduli for the effect of sample-rod interfaces. Examples of the application are given for homogeneous, isotropic polymer samples and a natural rock sample.
\end{abstract}




\section{INTRODUCTION}

Seismic properties of geological materials can depend on frequency, thus, laboratory measurements should be performed at field-employed frequencies. Unfortunately, laboratory measurement of lowfrequency $(<10 \mathrm{kHz})$ seismic properties is a difficult task with traditional ultrasonic equipment and limited sample size. Quasi-static techniques ${ }^{1}$ allow measurements of low-frequency seismic properties continuously from DC to up to several hundreds of hertz. However, such measurements are difficult and not commonly performed, because the technique requires accurate measurements of very small strains, and unwanted resonance can interfere with the measurements at high frequencies Resonant column tests ${ }^{2}$ allow measurements at tens of hertz, but the tests are usually conducted on unconsolidated sand and soil under low confining stresses. Conventional resonant bar tests ${ }^{3}$ allow measuring seismic properties of rocks and sediments at intermediate frequencies (typically hundreds of hertz to several kilohertz) relatively easily. One major disadvantage of this technique, however, is that the test requires a long, slender sample, which is often difficult to obtain from deep rock/sediment or from weak and fractured geological formations. If the conventional resonant bar test is applied to the 5-to- $10 \mathrm{~cm}$ long cores that are typically available from such formations, the resonance frequency can be as high as tens of kilohertz.

Alternatively, a composite bar consisting of a jacketed rock or sediment core sample mediating a pair of metal extension bars (including seismic sources and sensors) can be used to achieve lower resonance frequencies of the system than the sample itself. The reduction of the resonance frequency increases for longer length and larger mass of the extension bars. The basic principle of this technique is the same as for Tittmann ${ }^{4}$, who proposed the use of additional mass in resonant bar tests for reducing resonance frequencies. The geometry of this experimental setup is the same as the split Hopkinson pressure bar test ${ }^{5}$ used primarily for large-strain, dynamic impact experiments. For this reason, we call this technique the “Split Hopkinson Resonant Bar (SHRB)" test.

In the following, we will first describe the testing methodology of the SHRB test, then our experimental setup and the measurement procedures. The principles of numerical inversion for elastic moduli and seismic velocities and attenuation from laboratory measurements will also be discussed, along 
with necessary corrections to the results for removing measurement artifacts. Following the description of the method, we will show applications of the SHRB test. The first example will examine the accuracy of the technique when applied to isotropic, homogeneous polymers (acrylic and polycarbonate) with independently measured material properties. The second example uses a rock sample (Berea sandstone) that is injected with brine and subsequently drained. Finally, in the conclusion, we will summarize the strengths and weaknesses of the SHRB test.

\section{METHOD}

SHRB tests are applied in two steps. First, resonance frequencies and attenuation of the entire system (extension bars + sample) are measured in the laboratory. Next, using these measurements as an input, numerical inversion is performed to determine the sample's Young's modulus $E$ and shear modulus $G$, including the related attenuation (damping) parameters. These moduli can be used to determine the seismic velocities and attenuation.

\section{A. Experiment}

Our current SHRB setup uses ASTM 316 stainless steel bars with a diameter of $3.75 \mathrm{~cm}$ (1.5 inches) and a length of $40.6 \mathrm{~cm}$ (16 inches). At the end of each bar, either a source unit or a receiver unit is attached for seismic measurements [Fig. 1(a)]. The source unit houses both compression and polarized shear piezoceramics (Channel Industries C5500), and the receiver houses three miniature accelerometers (Endevco 27AM1-100, 258A-100). An axial accelerometer measures the longitudinal motions, and the remaining two accelerometers measure torsion motions. The polarization directions of the two are aligned so that the subtraction of measured signals results in enhancement of torsion motions, while contamination from unwanted flexural motions and electrical noise is reduced. 
For measuring seismic properties, a cylindrical core is placed between the extension bars. The diameter of the core is the same as the bars. The core is jacketed with a thin, PVC heat-shrink jacket (jackets with a thickness of $165 \mu \mathrm{m}$ and $500 \mu \mathrm{m}$ were used, depending on the stiffness of the sample) (Figure 1b). For hard, consolidated rock samples, thin lead foils are placed at the sample-rod interfaces to assure good mechanical coupling. Also, pore fluids can be injected and extracted during an experiment through ports in the extension bars, under controlled temperature and confining pressure.

Once assembled, the entire system, consisting of the extension bars, source, receiver, and the jacketed core sample, is suspended by steel springs within a tubular cage (Figure.1c). This entire assembly is inserted into a pressure vessel within which confining pressure is applied hydrostatically via compressed nitrogen gas. The seismic source is driven by either chirp or random signals with a desired range of frequencies, amplified by a broad-band high-voltage amplifier (Krhon-Hite 7602M). Signals measured by the accelerometers are conditioned (PCB Piezotronics ICP signal conditioner/amplifier), then analyzed by an FFT analyzer (ONO SOKKI CF-6400), which also generates the input signals.

For a given combination of temperature, confining pressure, and pore pressure, both compression mode ( $E$ mode) and torsion mode ( $G$ mode) vibrations are measured. The basic principle of the measurements is the same as the conventional resonant bar tests. Resonance frequencies are determined from the peak frequency of the amplitude or power spectra, and the attenuation from the half-power width of the peak (Figure 2).

\section{B. Numerical modeling and inversion}

Experimentally measured resonance frequencies and attenuation are for the resonance of the entire system and not for the sample alone. Unfortunately, a closed form solution relating the measured results directly to the sample properties is unavailable. For this reason, simple numerical modeling and inversion are conducted to determine the material properties of the sample.

\section{One-dimensional wave propagation model}


Numerical modeling is conducted assuming one-dimensional acoustic wave propagation for the lowest-order extension (Young's-modulus) and torsion (shear-modulus) modes. For the extension mode, this assumption is not valid - unless the Poisson ratio of the medium is zero-if the wavelength to bar diameter ratio approaches 1 . This is because the Poisson expansion of the bar results in radial stresses induced by the radial inertia. The frequency equation for the wave velocity in a homogeneous, finitediameter rod was obtained by Love ${ }^{6}$. An approximate solution, known as the Rayleigh-Love correction, is given by:

$$
\frac{\xi_{E}^{*}}{\xi_{E}}=\sqrt{1+\frac{1}{2}\left(\pi \nu \frac{d}{\lambda_{E}}\right)^{2}}
$$

where $\xi_{E}^{*}$ is the slowness of the extension mode wave, $\xi_{E}$ and $\lambda_{E}$ the theoretical slowness and wavelength for an infinitely slender rod, respectively; and $d$ and $v$ are the diameter and the Poisson ratio of the finite-diameter rod, respectively. The above equation can be used to correct for the dispersion of the extension waves. With SHRB tests, however, we are interested in the fundamental resonance mode, which has a much longer wavelength compared to the diameter of the sample and the rods. For this reason, the effect of velocity dispersion induced by the radial inertia is usually negligible, except for the case in which the velocity within the sample is extremely small compared to the rods.

For the one-dimensional system shown in Figure 3, we obtain a system of equations by requiring the continuity of wave-induced displacement and stress at the boundaries. The resulting equations are:

$$
\begin{aligned}
& U_{n}^{+} e^{i \omega \xi_{n} h_{n}}+U_{n}^{-} e^{-i \omega \xi_{n} h_{n}}=U_{n+1}^{+}+U_{n+1}^{-}(n=0,1), \\
& i \omega \xi_{n} K_{n}\left(U_{n}^{+} e^{i \omega \xi_{n} h_{n}}-U_{n}^{-} e^{-i \omega \xi_{n} h_{n}}\right)=i \omega \xi_{n+1} K_{n+1}\left(U_{n+1}^{+}-U_{n+1}^{-}\right)(n=0,1), \\
& i \omega \xi_{0} K_{0}\left(U_{0}^{+}-U_{0}^{-}\right)=-\omega^{2} M_{\text {source }}\left(U_{0}^{+}+U_{0}^{-}\right)+\Delta, \\
& i \omega \xi_{2} K_{2}\left(U_{2}^{+} e^{i \omega \xi_{2} h_{2}}-U_{2}^{-} e^{-i \omega \xi_{2} h_{2}}\right)=+\omega^{2} M_{\text {receiver }}\left(U_{2}^{+} e^{i \omega \xi_{2} h_{2}}+U_{2}^{-} e^{-i \omega \xi_{2} h_{2}}\right) .
\end{aligned}
$$

For the extension mode, $U$ refers to the axial displacement at the boundaries, $K$ the axial rigidity (=Young's modulus $\times$ area of the bar cross section), and $\xi$ the complex bar wave (Young's modulus 
wave) slowness. The subscripts indicate each segment of the system and the superscripts either forward $(+)$ or backward (-) propagating waves. For the torsion mode, these symbols are replaced by the rotational angle around the axis, torsional rigidity (=shear modulus $\times$ torsion constant or polar moment of inertia), and the torsion wave slownesses, respectively. The effect of the source and receiver units for each mode is modeled using a point mass ( $M$, for extension mode) or an angular mass $\left(M d^{2} / 8\right.$, for torsion mode) at the ends of the bar, which is indicated by $M_{\text {source }}$ and $M_{\text {receiver }}$. Finally, $\Delta$ provides either unit force (for extension mode) or torque (for torsion mode) excitation to the system. The above equations are solved for the displacements as a function of frequency. Using these results, the frequency response of the displacement at the receiver location is computed by

$$
U_{\text {receiver }}=U_{2}^{+} e^{i \omega \xi_{2} h_{2}}+U_{2}^{-} e^{-i \omega \xi_{2} h_{2}}
$$

Once a complex-valued frequency response curve is computed for a desired range of frequency including the fundamental-mode resonance, the resonance frequency and the attenuation are determined numerically, using the same method as in the laboratory measurements.

\section{Numerical inversion}

The one-dimensional wave propagation model in the previous section computes resonance frequency and attenuation for a specific combination of model sample properties. We use this model repeatedly to invert for the properties of the sample itself. Because wave propagations for extension and torsion modes are independent from each other, inversion for each elastic modulus ( $E$ and $G$ ) can be conducted separately.

The basic steps of the inversion are show in Figure 4. Although a variety of techniques could be used to correct the model elastic moduli at each iteration shown in the flowchart, the following method is used for simplicity. First, we express a complex elastic modulus $M$ via two real, positive numbers, as in $M \equiv M_{\text {real }}(1-2 i a)$. Here, $M_{\text {real }}$ is the real part of the modulus $M$ and $a$ is the attenuation coefficient. These two parameters are updated iteratively by multiplying the following correction factors: 


$$
\begin{aligned}
& C_{\text {Mreal }}=(1+r)^{2}, r \equiv 2\left(f_{\text {obs }}-f_{\text {comp }}\right) /\left(f_{\text {obs }}+f_{\text {comp }}\right), \\
& C_{a}=1+s, s \equiv 2\left(a_{o b s}-a_{\text {comp }}\right) /\left(a_{o b s}+a_{\text {comp }}\right),
\end{aligned}
$$

where $f$ 's are the resonance frequencies. The subscript $o b s$ indicates laboratory measurements and comp the numerically computed quantities. Values of both $r$ and $s$ are restricted between -0.9 and 0.9 for stability. Note that the correction factor in Equation (7) is squared, because the actual correction is applied to the slowness of the sample (obtained from the square root of the modulus) rather than the modulus,

\section{Corrections}

The numerical inversion determines complex-valued Young's and shear moduli of the sample in the one-dimensional model, which results in the same frequency and attenuation as the experiment. However, these are still not the actual properties of the tested material. This is because the experimental results are affected by three-dimensional effects of the sample jacket. In addition, the friction between the sample and the bar results in heterogeneous stress distribution near the interface. The errors in the inverted moduli must be corrected for these artifacts.

\section{Jacket correction}

SHRB tests use a gas-impermeable jacket for applying confining pressure and for holding pore fluid in the sample. For compliant samples and samples with small attenuation, this jacket can change the apparent elastic and attenuation properties of the sample.

For low-frequency torsion wave propagation, $\mathrm{Lai}^{7}$ computed the effective shear modulus $\bar{G}$ and the related density $\bar{\rho}_{G}$ of an infinite jacketed (clad) bar by

$$
\begin{aligned}
& \bar{G}=G+G_{j}\left[\left(1+\frac{t}{a}\right)^{4}-1\right] \approx G+G_{j} \cdot 4 \frac{t}{a}, \\
& \bar{\rho}_{G}=\rho+\rho_{j}\left[\left(1+\frac{t}{a}\right)^{4}-1\right] \approx \rho+\rho_{j} \cdot 4 \frac{t}{a},
\end{aligned}
$$


where $t$ is the jacket thickness and $a$ the radius of the bar (not to be confused with the attenuation coefficient). We assume that $t / a<<1$. The subscript $j$ indicates jacket properties. Note that the above effective modulus and density are defined for the sample radius rather than the outer radius of the jacket. The slowness of the wave within the sample is given by

$\xi_{G}=\sqrt{\bar{\rho}_{G} / \bar{G}}$

For extension mode vibrations, Lai also gives the Young's modulus of a composite bar

$$
\bar{E}=E+E_{j}\left[\left(1+\frac{t}{a}\right)^{2}-1\right] \approx E+E_{j} \cdot \frac{2 t}{a} .
$$

This result, however, is not accurate if the sample and the jacket have different Poisson ratios. Assuming that the interface between the jacket and the sample is "welded," the effective elastic properties of this composite bar can be computed by (the derivation is shown in Appendix A)

$$
\bar{E} \approx E+E_{j} \frac{2 t}{a} \frac{1-v^{2}}{1-v_{j}^{2}} .
$$

The related density of the medium is given by

$$
\bar{\rho}_{E}=\rho+\rho_{j}\left[\left(1+\frac{t}{a}\right)^{2}-1\right] \approx \rho+\rho_{j} \cdot 2 \frac{t}{a}
$$

Using these results, the slowness of the composite medium is

$$
\xi_{E}=\sqrt{\bar{\rho}_{E} / \bar{E}}
$$

The complex elastic moduli of the jacket are determined from an independent SHRB test using only a jacket without a sample.

\section{Interface correction}

Unlike the conventional resonant bar test, friction at the interfaces between a sample and extension rods in SHRB tests can introduce a non-1D stress state. This stress affects estimation of the Young's 
modulus from measured extension-mode resonances if a one-dimensional model is used to interpret the results. To keep the stress within a sample 1D, high-stress split Hopkinson pressure bar (SHPB) tests either lubricate the interfaces or use a very short sample, to keep the stress and strain within the sample uniform for nonlubricated or bonded interfaces. ${ }^{8}$

In SHRB tests, even small changes in resonance frequency and attenuation caused by a viscous lubricant can significantly affect the accuracy of the determined elastic moduli and attenuation. Therefore, a stiff, "welded" interface is preferred, which provides better-known boundary conditions for the numerical modeling. For stiff samples with smooth surfaces, this is ensured by placing a piece of thin, soft-metal foil at the interface, which deforms plastically and fills the small gaps and surface roughness once the confining pressure is applied to the sample. In contrast, for soft and unconsolidated media under confining stress, no foils are necessary. This is because frictional locking prevents the slip at the interface when small, dynamically induced shear stress is applied-a mechanism similar to the small-strain elastic deformation of fractured and/or granular rocks. ${ }^{9-11}$

The disadvantage of choosing a "welded" interface is that a measured Young's modulus of a sample must be corrected for the sample geometry (sample length to diameter ratio) and the material properties themselves (with respect to the elastic properties of the metal bars). The effect of the friction at the interface causing non-1D stress within a sample is demonstrated in Figure 5, using an axisymmetric, elasto-static finite element model. For this analysis, the circular bar's Young's modulus $E_{b}=200 \mathrm{GPa}$, Poisson's ratio $v_{b}=0.3$, sample Young's modulus $E_{s}=5 \mathrm{GPa}$, Poisson's ratio $v_{s}=0.33 ; a$ is the radius of both sample and the bar., Note that the finite element modeling is conducted for only one quarter of the whole system because of the symmetry. For this reason, the actual length of the sample and the whole system being modeled here are $2 a$ and $8 a$, respectively.

An analytical expression is useful for determining the true sample Young's modulus from an apparent Young's modulus determined from the numerical inversion. For deriving such an expression, from observations of the finite element modeling results in Fig.5, we first assume that the conical regions of friction-induced stresses exist on the both sides of the sample-bar interface, and that a uniform lateral 
strain is induced by the axial vibration in these regions (Fig.6). We also assume that the size and geometry of these regions are identical for both metal bar and the sample. This assumption leads to lateral stresses with equal magnitude but opposite sign within the conical regions on both metal and sample sides. The rest of the core and the metal bar is assumed to be at uniaxial stress state (with no vibration-induced lateral stress). Next, we further simplify the model by assuming that the apparent axial compression of the core can be given by a sum of the axial compression of two layers - one with the given lateral strain of the metal rods and the other under a uniaxial stress state — and an excess deformation within the metal bar. This simplification allows us to derive an expression for an apparent Young's modulus of the sample, as a function of the extension bar and the sample's Young's moduli, shear moduli (or Poisson's ratios), sample diameter, sample length, and the angle of the cone for the zone affected by the friction. (This derivation is shown in Appendix B.) The effective Young's modulus considering the interface friction effect can be computed via

$$
\bar{E}_{s}=\frac{E_{s}}{1-\Delta}
$$

where

$$
\Delta \equiv \frac{2 h}{H} \frac{\left(v_{s}-\frac{E_{s}}{E_{b}} v_{b}\right)^{2}}{1-v_{s}+\frac{E_{s}}{E_{b}}\left(1-v_{b}\right)}, h=\frac{2}{3} a \tan \theta .
$$

The angle $\theta$ is the slope of the conical, laterally constrained zones, which is used as an adjustable parameter. From a series of elasto-static finite-element simulations, it was found that an angle of $\sim 27.5^{\circ}$ was adequate for matching the effective Young's moduli from the theory and the numerical simulations for a wide range of sample elastic properties and geometry. (See Appendix C-differences between the true Poisson ratios and corrected Poisson ratios using the idealized model were minimized.) Figure 7 shows Poisson ratios computed from the apparent Young's moduli as a function of the sample's Young's modulus, Poisson's ratio, and the length-to-diameter (H/D) ratio. Predictions using the equations (16) and 
(17) (shown in solid curves) are compared to the finite element model (discrete dots), showing very good agreement. As both models indicate, the differences between the apparent and true Poisson's ratio become large for smaller H/D's, smaller sample Young's moduli, and larger true Poisson ratios.

Note that when $H / D<\tan \theta(\sim 0.52)$, the constrained zones can overlap. However, although the derivation of the correction equation did not consider this effect, numerical simulations indicated that the above equations perform well even for samples with small $H$ to $D$ ratios (Figure 7).

\section{EXAMPLES AND DISCUSSIONS}

In this section, we will examine some characteristics of the SHRB test through examples from two sets of laboratory experiments. In the first example, we will examine the accuracy of the method using homogeneous and isotropic polymer (acrylic and polycarbonate) cylinders. In the second example, we will apply the technique to a natural sandstone sample during injection and drainage of fluid (brine).

\section{A. Polymer Cylinders}

\section{Measurements and the results of inversion}

To examine the accuracy of the complex elastic moduli determined by the SHRB test, a series of laboratory measurements was conducted using acrylic and polycarbonate cylinders (obtained from McMaster-Carr) with a range of length $(\mathrm{H}=0.95,1.9,3.8,7.6$, and $11.4 \mathrm{~cm})$. The experiments were conducted with a confining stress of $8 \mathrm{MPa}$. An example of measured frequency response curves for an acrylic sample $(\mathrm{H}=7.6 \mathrm{~cm})$ is shown in Figure 8a. For comparison, frequency response curves from a conventional resonant bar test using a long bar $(\mathrm{H}=92 \mathrm{~cm}, \mathrm{D}=3.75 \mathrm{~cm})$ are also shown in Figure $8 \mathrm{~b}$.

Unlike the conventional resonant bar test, resonance peaks of the SHRB test are not equally spaced. Typically, the fundamental mode of SHRB tests is well isolated from other resonance peaks, and the higher-order mode peaks tend to cluster together by a group of two or more peaks. 
The real part of the Young's moduli $E$ and shear moduli $G$, and their attenuation, $a_{E}=-1 / 2 \cdot \operatorname{Im}(E) / \operatorname{Re}(E)$ and $a_{G}=-1 / 2 \cdot \operatorname{Im}(G) / \operatorname{Re}(G)$, respectively, were determined by the inversion. The results are shown in Figure 9. The open squares and triangles, both connected by a solid line, refer to the quantities related to $E$ and $G$, respectively. Note that increasing resonance frequencies in the plot correspond to shorter sample lengths for the same material type. The "true" material properties near the measurement frequencies were determined from conventional resonance tests using long circular bars $(\mathrm{H}=92 \mathrm{~cm}, \mathrm{D}=3.75 \mathrm{~cm})$ of the same material. These results are shown by filled circles for $E$ mode and filled triangles for $G$ mode, respectively. For comparison, Figure 9 also includes the results for the Young's modulus without applying the interface correction (shown in open circles connected by broken lines),

Conventional ultrasonic transmission tests were also used to determine high-frequency Young's and shear moduli, from $\mathrm{P}$ and $\mathrm{S}$-wave velocities of the polymer samples with lengths $\mathrm{H}=0.95,1.9$, and $3.8 \mathrm{~cm}$ (indicated at the right edge of the plots in Figure 9). The center frequencies of the measured waves for polycarbonate samples were $\sim 550 \mathrm{kHz}$ for $\mathrm{P}$ waves and $200-250 \mathrm{kHz}$ for $\mathrm{S}$ waves. For acrylic samples, the frequencies were $590-660 \mathrm{kHz}$ for $\mathrm{P}$ waves and $280-450 \mathrm{kHz}$ for $\mathrm{S}$ waves. Both Young's and shear moduli of the samples from the ultrasonic measurements are $\sim 15 \%$ (for polycarbonate) and $\sim 12 \%$ (for acrylic) larger than the resonance tests, possibly because of the frequency-dependent properties of the materials. Attenuations from the ultrasonic measurements are not shown here because the results using the transmission tests are usually unreliable.

\section{Discussion, tests on polymer samples}

The differences between the corrected and uncorrected results in Figure 9 demonstrate that the interface correction can be very large for short samples (with higher resonance frequencies) and high Poisson's ratio materials (Poisson ratios for polycarbonate and acrylic are $\sim 0.4$ and 0.33 , respectively). Generally, the elastic moduli of the samples determined via the SHRB test are in very good agreement 
with the conventional resonant bar tests. Also, in the examined frequency range, moduli (or velocity) dispersion is very small. Attenuation also shows good agreement, although the differences between the two tests are greater.

If the frequency dependence of moduli can be ignored between $\mathrm{E}$ and G-mode resonance frequencies, we can determine P-wave velocity and attenuation from a SHRB test on a single sample. Using complex elastic moduli $E$ and $G$, the P-wave modulus is $H=G(4 G-E) /(3 G-E)$. P and S-wave velocities are therefore $V_{P}=\sqrt{\operatorname{Re}(H) / \rho}$ and $V_{S}=\sqrt{\operatorname{Re}(G) / \rho}$, respectively, where $\rho$ is the density of the sample. The related attenuation is computed by $a_{P}\left(=1 / 2 Q_{P}\right)=-(1 / 2) \cdot \operatorname{Im}(H) / \operatorname{Re}(H)$ and $a_{G}\left(=1 / 2 Q_{S}\right)=-(1 / 2) \cdot \operatorname{Im}(G) / \operatorname{Re}(G)$. Note that $Q_{P}$ and $Q_{S}$ are the seismic quality factors. The determined velocities and attenuation shown in Figure 10 are compared to the conventional resonant bar tests, including the velocities from ultrasonic measurements. The results from the conventional tests were obtained by fitting smooth curves to the measured resonance frequencies and attenuation as a function of frequency. Compared to $E$ and $G$ in Figure 9, P-wave velocities show relatively large errors. Apart from possible errors from the frequency dependencey of $E$ and $G$, this is primarily because even very small errors in the moduli can be amplified when computing $H^{12}$. The error becomes greater as the Poisson ratio of the sample approaches 0.5 , because of the relationship

$$
H=E \frac{1-v}{(1-2 v)(1+v)}=2 G \frac{1-v}{1-2 v} .
$$

As Figure 10 shows, the disagreement between the attenuation results is even more severe partly because of the higher noise in $a_{E}$ and $a_{G}$. For small attenuations $\left(a_{E}, a_{G} \square 1\right)$, P-wave (or $H$-modulus) attenuation is given by the following expression modified from the Winkler and Nur's result ${ }^{13}$

$a_{P} \approx a_{E}+\frac{2 v(2-v)}{(1-v)(1-2 v)}\left(a_{E}-a_{G}\right)$

Note that from Fig.10 the conventional resonant bar tests on a polycarbonate sample even resulted in unphysical, negative attenuation values. Amplifications of errors in computing $a_{P}$ are also to blame here, 
because for a polycarbonate sample with $v=0.4$, Equation (19) amplifies the errors by a factor of $\sim 12$.

Turner ${ }^{14}$ also pointed out the extreme sensitivity of complex bulk modulus determined from Young's and shear moduli, to experimental errors and the frequency dependence. In the current experiment, the relatively large errors in the attenuation of the conventional resonant bar measurements may also be attributed to the energy loss within the glue attaching the seismic sources to the bar surface. Reduced attenuation was observed during conventional resonant bar tests when the temperature of source and receiver interfaces was lowered, which indicated that the properties of the glue used to attach them to the sample affected the results. Also, for the SHRB tests, the surfaces of the samples had to be made flat with extreme care. Still remaining, finite compliance in the interface may have contributed to small but finite errors in the measurements, which resulted in the differences in the P-wave velocities. Interestingly, the attenuation results from the SHRB tests for each polymer type show only small variations among measurements at different frequencies, indicating better robustness of this method than the conventional resonant bar test. This may be because the epoxy-potted sources of the SHRB setup are more firmly attached to the surface of the metal extension rod.

\section{B. Sandstone core}

In the second example, we will examine changes in the seismic velocities and attenuation of a natural rock sample (Berea sandstone), during injection into and subsequent drainage of brine $(0.5 \mathrm{M} \mathrm{KCl}$ aqueous solution) from its pore space.

\section{Experiment and the results of inversion}

A dry, homogeneous Berea sandstone core (porosity $21.2 \%$, permeability $620 \mathrm{mD}$, core diameter 3.81 $\mathrm{cm}$, and length $6.22 \mathrm{~cm}$ ), flushed with supercritical $\mathrm{CO}_{2}$ prior to the experiment, was first filled with $\mathrm{CO}_{2}$ gas at $0.69 \mathrm{MPa}$, under a confining stress of $4.14 \mathrm{MPa}$. Subsequently, SHRB tests were conducted while brine $(0.5 \mathrm{M} \mathrm{KCl}$ aqueous solution) was injected into and then drained from the sample. The injection was done by increasing the injection pressure by steps, while maintaining a differential pressure of 3.45 
$\mathrm{MPa}$ between the confining stress and the pore fluid pressure. As the pore pressure was increased, the $\mathrm{CO}_{2}$ in the pore space dissolved into the brine. Once the sample was fully saturated with brine, both pore and confining pressures were reduced to allow a part of the dissolved $\mathrm{CO}_{2}$ gas to come out of the pore fluid, driving the fluid out of the sample. The differential pressure was maintained at $3.45 \mathrm{MPa}$. Once the pore pressure was reduced to the atmospheric pressure, the pore fluid was forced to drain by flowing compressed air through the sample. Note that imbibing brine into an initially dry sandstone core results in a distribution of unsaturated and nearly-saturated zones separated by a well-defined invading front. In contrast, draining the brine by de-gassing of dissolved gas and by flowing compressed air should result in more evenly distributed unsaturated phase. The saturation of the sample was determined from the amount of the brine injected and extracted out of the sample, relative to the sample pore volume.

During the experiment, the resonance frequencies changed within a range of $1.63-1.76 \mathrm{kHz}$ for the extension mode and $1.05-1.14 \mathrm{kHz}$ for the torsion mode. Inversions were performed, and $\mathrm{P}$ and $\mathrm{S}$-wave velocities and attenuation were determined, assuming that the dispersion of the moduli was small between the resonance frequencies of the two modes (Figure 11). The Poisson ratio of the sample varied between 0.17 (dry) and 0.34 (fully saturated). Both P and S-wave velocities initially decreased with increasing saturation, with the rate of decrease higher for saturations below $\mathrm{S}=45 \%$. At very high saturations, the Pwave velocity showed rapid increases, reaching the maximum at $100 \%$ saturation. In contrast, P and Swave attenuations gradually increased up to $\mathrm{S}=45 \%$. For higher saturations, S-wave attenuation did not change. However, P-wave attenuation exhibited a peak immediately before reducing to a much smaller value at full saturation.

\section{Discussion, sandstone test}

Some of the initial decreases in velocities with increasing saturation can be attributed to increases in the density of the sample, as the classical Gassmann's theory predicts. ${ }^{15}$ The theory also predicts the observed rapid increases in P-wave velocity near full saturation. However, the observed changes below $\mathrm{S}=45 \%$ are too large to explain using the density effect alone and may be attributed to initial chemical 
softening of grain contacts ${ }^{16}$. For this reason, the velocity predictions using Gassmann models in Figure 11 are made based upon velocities measured at $\mathrm{S}=50 \%$. A good agreement is found between the model and the experiment for the S-wave velocities for higher saturations. In contrast, although the P-wave velocity also shows a good agreement for $S=50-80 \%$, the model underestimates the velocity for higher saturations.

This discrepancy is larger during the injection cycle than the drainage cycle because P-wave velocity is higher during the injection. Also, increases in P-wave attenuation for $\mathrm{S}>50 \%$ are larger for the injection cycle than the drainage cycle, with the maximum attenuation occurring at a slightly lower saturation. This hysteretic behavior is attributed to the differences in the relative size of the fluid (or gas) patches compared to the wavelength of diffusing pressure waves within the fluid-saturated pores ${ }^{12}$. During the injection cycle, the relaxation of the wave-induced fluid pressure is mostly limited to near the invading front, resulting in well-segregated distribution of gas and fluid. In contrast, during the drainage cycle, the pore pressure relaxation happens in a larger volume in the sample, because of more uniformly distributed, de-gassing induced gas bubbles. Because of this effect, $\mathrm{P}$-wave velocities during the drainage are in better agreement with the predictions, indicating that the measured seismic response is closer to the "relaxed", low-frequency limit assumed by the Gassmann model. Also, a maximum in the attenuation indicates that the characteristic size of the fluid patch is close to the diffusing pressure wave wavelength for the frequency of the test. Using a small Berea sandstone sample similar to our experiment, Yin et al. ${ }^{17}$ showed that this peak became less pronounced with decreasing frequency, and disappeared for measurements conducted at $100 \mathrm{~Hz}$ (This result was presented for the attenuation of bar [Young's modulus] waves).

\section{CONCLUSIONS}

Split Hopkinson Resonant Bar (SHRB) tests allow us to measure seismic velocity and attenuation of small rock and sediment cores in the laboratory at frequencies near $1 \mathrm{kHz}$. Because the SHRB test is 
essentially a resonant bar method, it is capable of measuring intrinsic (rather than scattering-induced) attenuation of the sample. Unlike the conventional resonant bar tests, however, SHRB tests require a correction of obtained Young's modulus (or Poisson's ratio) for the artifact caused by the friction between the sample and the extension bars. The presented correction method for an apparent Young's modulus is also applicable to other laboratory techniques such as the quasi-static method, involving an interface between a sample and a stiff platen.

As with other low-frequency techniques measuring Young's modulus and shear modulus (or Poisson ratio) for determining P-wave properties, velocity, and particularly attenuation of $\mathrm{P}$ waves can have a significant amount of error if the Poisson ratio of the sample is high. Therefore, great care must be taken when testing high Poisson ratio materials such as plastics, rubbers, and fluid-saturated rocks. In spite of these errors, SHRB tests can also be used as a sensitive spectroscopic technique for measuring small changes in material properties rather than for determining absolute properties. As the examples indicate, the SHRB test is suited for time-lapse monitoring of seismic velocities and attenuation in the laboratory during fluid injection in rock and sediment samples.

Additionally, because SHRB tests measure only the fundamental resonance modes, it is difficult to examine the frequency dependence of sample moduli if only a single sample is available. Although it is possible to use higher-order modes to make measurements at multiple frequencies, such modes will require development of more sophisticated numerical models reflecting the actual geometry and material properties of the experimental setup. Further, resonance frequencies for the extension and torsion modes are always different, which makes the velocity and attenuation of $\mathrm{P}$ wave inaccurate if the dispersion of the Young's and shear moduli is large. This problem can be solved if multiple similar samples with different core lengths are available, which provides a range of resonance frequencies. Another possible solution is to measure the lateral expansion of the sample from extension mode resonance, and compute $\mathrm{P}$ wave velocity and attenuation from Young's modulus and Poisson's ratio determined at a single frequency. This, however, will require very careful measurements of vibration amplitude and phase, similar to the quasistatic measurement of material properties. ${ }^{18}$ 
Lastly, the SHRB test can be easily conducted during concurrent x-ray CT imaging of the sample within a confining cell, as has been done with conventional resonant bar tests. ${ }^{12,19}$ Such experiments are currently being conducted to correlate gas/fluid distribution in the sample to the seismic properties of porous geological materials.

\section{ACKNOWLEDGMENTS}

This research was supported by the Office of Science, Office of Basic Energy Sciences, Division of Chemical Sciences of the U.S. Department of Energy, and by the Assistant Secretary for Fossil Energy, Office of Natural Gas and Petroleum Technology, NGOTP program and GEO-SEQ Program, through the National Energy Technology Laboratory, under the U.S. DOE, Contract No. DE-AC02-05CH11231. 


\section{APPENDIX A: JACKET CORRECTION FOR SAMPLE YOUNG'S MODULUS}

We consider the a cylindrical sample of radius $a$ with a jacket of outer radius $b$ and a thickness $t(=b$ $a$ ). Superscripts $(j)$ and $(s)$, and subscripts $j$ and $s$, indicate jacket and sample, respectively. From the standard plane strain elasticity solutions (Lamé's equations), the radial and tangential (hoop) stresses at the jacket-sample interface are

$$
\sigma_{t}^{(j)}=-\frac{b^{2}+a^{2}}{b^{2}-a^{2}} \sigma_{r}^{(j)} \equiv-B \sigma_{r}^{(j)} .
$$

Note that the axial stress is uniform within the jacket and within the sample. The strain components within the jacket and sample are

$$
\begin{aligned}
& \varepsilon_{z}^{(s)}=\frac{1}{E_{s}}\left(\sigma_{z}^{(s)}-2 v_{s} \sigma_{r}^{(s)}\right) \\
& \varepsilon_{z}^{(j)}=\frac{1}{E_{j}}\left[\sigma_{z}^{(j)}-v_{j}\left(\sigma_{r}^{(j)}+\sigma_{t}^{(j)}\right)\right]=\frac{1}{E_{j}}\left[\sigma_{z}^{(j)}-v_{j} \sigma_{r}^{(j)}(1-B)\right] \\
& \varepsilon_{t}^{(s)}=\frac{1}{E_{s}}\left[\sigma_{r}^{(s)}-v_{s}\left(\sigma_{r}^{(s)}+\sigma_{z}^{(s)}\right)\right]=\frac{1}{E_{s}}\left[-v_{s} \sigma_{z}^{(s)}+\left(1-v_{s}\right) \sigma_{r}^{(s)}\right] \\
& \varepsilon_{t}^{(j)}=\frac{1}{E_{j}}\left[\sigma_{t}^{(j)}-v_{s}\left(\sigma_{r}^{(j)}+\sigma_{z}^{(j)}\right)\right]=\frac{1}{E_{j}}\left[-B\left(1+v_{s}\right) \sigma_{r}^{(j)}-v_{s} \sigma_{z}^{(j)}\right]
\end{aligned}
$$

We assume that the interface between the jacket and the sample is welded and the radial stress is continuous.

$$
\begin{aligned}
& \varepsilon_{z}^{(s)}=\varepsilon_{z}^{(j)} \\
& \varepsilon_{t}^{(s)}=\varepsilon_{t}^{(j)} \\
& \sigma_{r}^{(s)}=\sigma_{r}^{(j)}
\end{aligned}
$$

These eight equations can be used to express axial stresses and strains only as a function of the radial stress on the interface:

$$
\left[\begin{array}{c}
\sigma_{z}^{(s)} \\
\sigma_{z}^{(j)}
\end{array}\right]=\frac{1}{v_{j}-v_{s}}\left[\begin{array}{c}
2 v_{s} v_{j}+v_{s}-1+\frac{E_{s}}{E_{j}}\left[B\left(v_{j} v_{j}-1\right)-v_{j} v_{j}-v_{j}\right] \\
\frac{E_{j}}{E_{s}}\left(2 v_{s} v_{s}+v_{s}-1\right)+\left[B\left(v_{s} v_{j}-1\right)-v_{s} v_{j}-v_{j}\right]
\end{array}\right] \sigma_{r}^{(s)},
$$


$\varepsilon_{z}=\frac{1}{E_{s}\left(v_{j}-v_{s}\right)}\left(2 v_{s} v_{s}+v_{s}-1+\frac{E_{s}}{E_{j}}\left[B\left(v_{j} v_{j}-1\right)-v_{j} v_{j}-v_{j}\right]\right) \sigma_{r}^{(s)}$.

The effective stress defined for the cross section of the sample is

$\bar{\sigma}_{z}=\sigma_{z}^{(s)}+\left[\left(\frac{b}{a}\right)^{2}-1\right] \sigma_{z}^{(j)}=\sigma_{z}^{(s)}+\frac{2}{B-1} \sigma_{z}^{(j)}$

By introducing Equations (A4) into Equation (A6), and then dividing the result by Equation (A5), the effective Young's modulus can be obtained. For small $t / a$ 's, neglecting second and higher-order terms of $t / a$ (Note that $B \rightarrow t / a$ for thin jackets),

$\bar{E}_{s} \equiv \frac{\bar{\sigma}_{z}}{\varepsilon_{z}} \rightarrow E_{s}+\frac{2 t}{a} E_{j} \frac{1-v_{s}^{2}}{1-v_{j}^{2}}$

\section{APPENDIX B: INTERFACE EFFECT ON THE SAMPLE YOUNG'S MODULUS}

We will derive an analytical equation predicting an apparent Young's modulus of the sample when the material properties and geometry of both sample and the extension bars are known. Consider the model geometry in Figure 6. Within the three sections indicated by $B, I$, and $I I$, stress state is assumed to be homogeneous. Also, we assume the axial stress $\sigma_{z}$ is uniform and continuous in the system. The strains within the section $B$ in the bar are

$$
\begin{aligned}
& \varepsilon_{r}^{(B)}=\frac{1}{E_{b}}\left[\left(1-v_{b}\right) \sigma_{r}^{(B)}-v_{b} \sigma_{z}\right], \\
& \varepsilon_{z}^{(B)}=\frac{1}{E_{b}}\left(\sigma_{z}-2 v_{b} \sigma_{r}^{(B)}\right) .
\end{aligned}
$$

Strains in the sample are 


$$
\begin{aligned}
& \varepsilon_{r}^{(I)}=\frac{1}{E_{s}}\left[\left(1-v_{s}\right) \sigma_{r}^{(I)}-v_{s} \sigma_{z}\right], \\
& \varepsilon_{z}^{(I)}=\frac{1}{E_{s}}\left(\sigma_{z}-2 v_{s} \sigma_{r}^{(I)}\right), \\
& \varepsilon_{z}^{(I I)}=\frac{1}{E_{s}} \sigma_{z} .
\end{aligned}
$$

Note that the subscripts on the material properties indicate $b \rightarrow$ bar and $s \rightarrow$ sample.

Assume that the radial stress within section B is equal in magnitude as section $I$, but has the opposite sign (i.e. $\sigma_{r}^{(B)}=-\sigma_{r}^{(I)}$ ). From the continuity of radial strain between sections $B$ and $I, \varepsilon_{r}^{(B)}=\varepsilon_{r}^{(I)}$, equations (B1) and (B2) result in

$$
\sigma_{r}^{(I)}=\frac{\frac{v_{s}}{E_{s}}-\frac{v_{b}}{E_{b}}}{\frac{1-v_{s}}{E_{s}}+\frac{1-v_{b}}{E_{b}}} \sigma_{z}
$$

The total strain within the sample including the excess strain within the bars is

$$
\bar{\varepsilon}_{z}=\frac{h}{H} \varepsilon_{z}^{(I)}+\left(1-\frac{h}{H}\right) \varepsilon_{z}^{(I I)}+\frac{h^{\prime}}{H}\left(\varepsilon_{z}^{(B)}-\varepsilon_{z 0}^{(B)}\right)
$$

Note that $\varepsilon_{z 0}^{(B)}=\sigma_{z} / E_{b}$ is the strain within the bars unaffected by the interface. Assuming sections $B$ and $I$ have the same geometry (i.e. $h^{\prime}=h$ ), and expressing the strains in equation (B4) via $\sigma_{z}$, the effective Young's modulus can be given by

$$
\bar{E}_{s} \equiv \frac{\sigma_{z}}{\bar{\varepsilon}_{s}}=\frac{E_{s}}{1-\Delta}
$$

where

$$
\Delta \equiv \frac{2 h}{H} \frac{\left(v_{s}-\frac{E_{s}}{E_{b}} v_{b}\right)^{2}}{1-v_{s}+\frac{E_{s}}{E_{b}}\left(1-v_{b}\right)}
$$

This result is used in Appendix C. 


\section{APPENDIX C: COMPUTATION OF A SAMPLE POISSON'S RATIO FROM AN EFFECTIVE POISSON'S RATIO}

Using the analytical model developed in Appendix B, the "true" Young's modulus of the sample is determined from a measured, apparent modulus. We will also examine the errors involved in this correction. The correction factor $\Delta$ in Appendix B can be written in the following form:

$$
\Delta=\frac{2 h}{H} \frac{v_{s}^{2}}{1-v_{s}}-\delta
$$

where

$$
\delta \equiv \frac{2 h}{H}\left[\frac{v_{s}^{2}}{1-v_{s}}-\frac{\left(v_{s}-\frac{E_{s}}{E_{b}} v_{b}\right)^{2}}{1-v_{s}+\frac{E_{s}}{E_{b}}\left(1-v_{b}\right)}\right] .
$$

Note that $\delta$ can be ignored if the Young's modulus of the sample is much smaller than the bars. The correction formula for the Young's modulus is

$$
\bar{E}_{s}=\frac{E_{s}}{1-\Delta} .
$$

Replacing the Young's modulus by the sample's shear modulus and Poisson's ratio,

$$
2 G_{s}\left(1+\bar{v}_{s}\right)=\frac{2 G_{s}\left(1+v_{s}\right)}{1-\frac{2 h}{H} \frac{v_{s}^{2}}{1-v_{s}}+\delta} .
$$

Note that the shear modulus is not affected by the interface effect. This equation can be written in the following form:

$$
f\left(v_{s}\right) \equiv\left[1-\left(1+\bar{v}_{s}\right) \frac{2 h}{H}\right] v_{s}^{2}-\left(1+\bar{v}_{s}\right) v_{s}+\bar{v}_{s}=-\left(1+\bar{v}_{s}\right)\left(1-v_{s}\right) \delta .
$$

Note that, if $\bar{v}_{s}$ is a real number, $f(0)=\bar{v}_{s} \geq 0$ and $f(1)=-\left(1+\bar{v}_{s}\right) \cdot 2 h / H<0$, which guarantees that the quadratic equation $f\left(v_{s}\right)=0$ has only one real-valued solution between 0 and 1 . Because the imaginary 
part of $\bar{v}_{s}$ is usually very small, this solution can be used as an initial value to solve the above equation iteratively via:

$f\left(v_{s}^{(n+1)}\right)=-\left(1+\bar{v}_{s}\right)\left(1-v_{s}^{(n)}\right) \delta^{(n)}, n=0,1,2, \ldots f\left(v_{s}^{(0)}\right)=0$

where $\delta^{(n)}$ is computed for $v_{s}^{(n)}$ and $E_{s}^{(n)}=2 G_{s}\left(1+v_{s}^{(n)}\right)$.

Using the apparent elastic moduli computed from the FEM simulations in Section C.2 as an input, we apply this inversion scheme to recover the "true" Poisson's ratios of the samples. The differences between the determined and "true" Poisson's ratios are shown in Figure 12. For the parameters examined in this example, the estimation errors for the Poisson's ratios are contained within $+/-0.01$. 


\section{REFERENCES}

${ }^{1}$ See, for example, the paper: J.W. Spencer, "Stress relaxation at low frequencies in fluid saturated rocs: attenuation and modulus dispersion", J. Geoph. Res., 86, B3, 1803-1812 (1981).

${ }^{2}$ See, for example, the paper: B. O. Hardin and F. E. Richart, Jr., J. Soil Mech. Found. Div., ASCE 89, 33 (1963)

${ }^{3}$ See, for example, the paper: Lucet, N., N.J. Rasolofosaon, and B. Zinszner, "Sonic properties of rocks under confining pressure using the resonant bar technique", J. Acoust. Soc. Am., 89(3), 980-990 (1991)

${ }^{4}$ Tittmann, B.R., "Internal friction measurements and their implications in seismic Q structure models of the crust", In The earth's crust, AGU Monograph, 20, J.G. Heacock (ed.), American Geophysical Union, Washington, D.C., 197-213 (1977).

${ }^{5}$ Kolsky H., "An investigation of the mechanical properties of materials at very high rates of loading”, Proc. Phys. Soc. Lond. B, 62, 676-700 (1949).

${ }^{6}$ Love, A.E., A treatise on the mathematical theory of elasticity, Dover Publications, New York, 289-291 (1944).

${ }^{7}$ Lai, J.-L., "Propagation of harmonic waves in a composite elastic cylinder", J. Acoust. Soc. Am., 49, 220-228 (1970).

${ }^{8}$ Bertholf, L.D. and Karnes, C.H., "Two-dimensional analysis of the split Hopkinson pressure bar system”, J. Mech. Phys. Solids, 23, 1-19 (1975).

${ }^{9}$ Cook, N.G.W., and Hodgson, K., "Some detailed stress-strain curves for rock", J. Geophys. Res., 70(12), 2883-2888 (1965).

${ }^{10}$ Walsh, J.B., "The effect of cracks on the uniaxial elastic compression of rocks", J. Geophys. Res., 70 (2), 399-411 (1965).

${ }^{11}$ Hilbert, L.B., Kwong, T.K., Cook, N.G.W., Nihei, K.T., and Myer, L.R., "Effects of strain amplitude on the static and dynamic non-linear deformation of Berea sandstone", In: Rock Mechanics Models and Measurements: Challenges from Industry, Nelson, P. and Laubach S. (eds), Proc. The $1^{\text {st }}$ North American Rock Mechanics Symposium, Balkema, Rotterdam, 487-504, (1994).

${ }^{12}$ Cadoret, T., D. Marion, and B. Zinszner, "Influence of frequency and fluid distribution on elastic wave velocities in partially saturated limestones”, J. Geophys. Res., 100, 154-160 (1995).

${ }^{13}$ Winkler, K.W. and A. Nur, "Pore fluids and seismic attenuation in rocks", Geophys. Res. Lett., 6, 1-4 (1979). 
${ }^{14}$ Turner, S., Mechanical Testing of Plastics, $2^{\text {nd }}$ ed., George Goodwin in association with The Plastics and Rubber Institute, Longman House, New York, 53 (1983).

${ }^{15}$ F. Gassmann, F., Vier. der Natur. Gesellschaft in Zürich, 96, 1 (1951); English translation is available from http://sepwww.stanford.edu/sep/berryman/PS/gassmann.pdf (Date last viewed 01/03/11).

${ }^{16}$ Murphy, W. F., III, K. W. Winkler, and R. L. Kleinberg, "Frame modulus reduction in sedimentary rocks: The effect of adsorption on grain contacts", Geophys. Res. Lett., 11(9), 805808 (1984).

${ }^{17}$ C.-S. Yin, M. L. Batzle, and B. J. Smith, Geophys. Res. Lett., 19, 1399 (1992)

${ }^{18}$ Batzel, M.L., D.-H. Han, and R. Hofmann, "Fluid mobility and frequency-dependent seismic velocity—Direct measurements", Geophysics, 71, N1-N9 (2006).

${ }^{19}$ See, for example, the paper: Cadoret, T., G. Mavko, and B. Zinszner, "Fluid distribution effect on sonic attenuation in partially saturated limestones", Geophysics, 63, 154-160 (1998). 
Figure 1. SHRB test setup

(a) Source and receiver units attached to the ends of the bars. These units were later potted with high-strength epoxy for protection.

(b) Jacketed sample core placed between steel extension bars

(c) Gas confining cell (top right) and a suspension cage (front) housing the sample-bar assembly

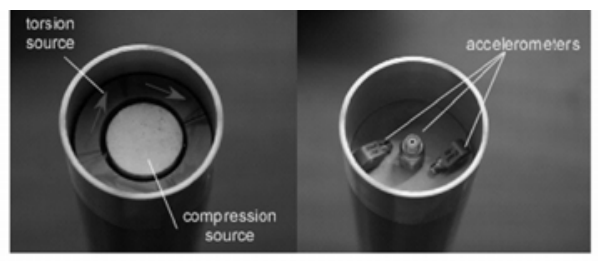

(a) Source and receiver units attached to the ends of the bars. These units were later potted with high-strength epoxy for protection.

(b) Jacketed sample core placed between steel extension bars

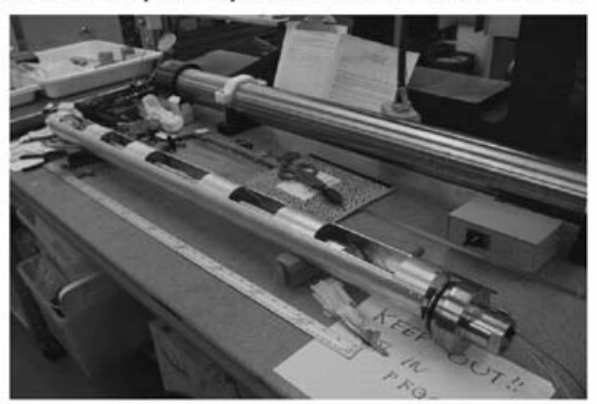

(c) Gas confining cell (top right) and a suspension cage (front) housing the sample-bar assembly

Figure 2. The half-power method. Seismic quality factor Q and damping coefficient $a$ can be computed from the resonance frequency and the width of the resonance peak.

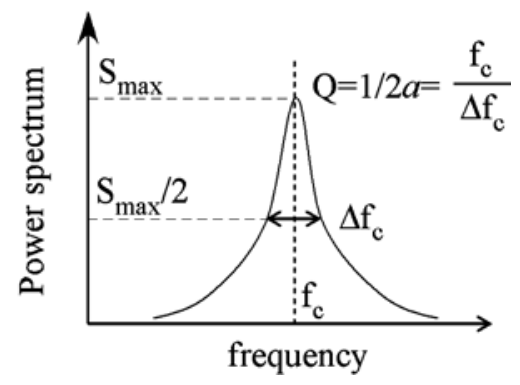


Figure 3. One-dimensional system used for modeling wave propagation within the SHRB

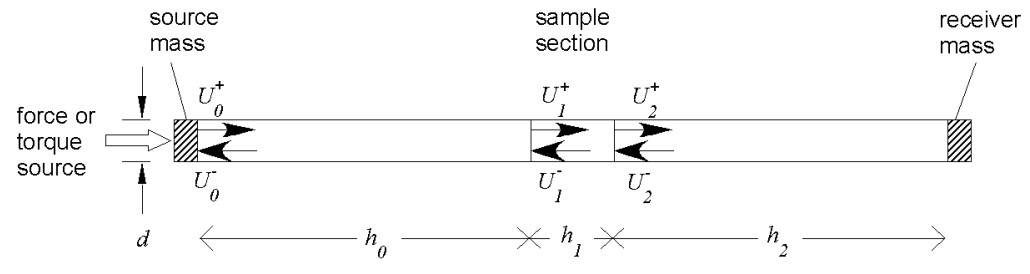

Figure 4. Flowchart for complex elastic modulus inversion

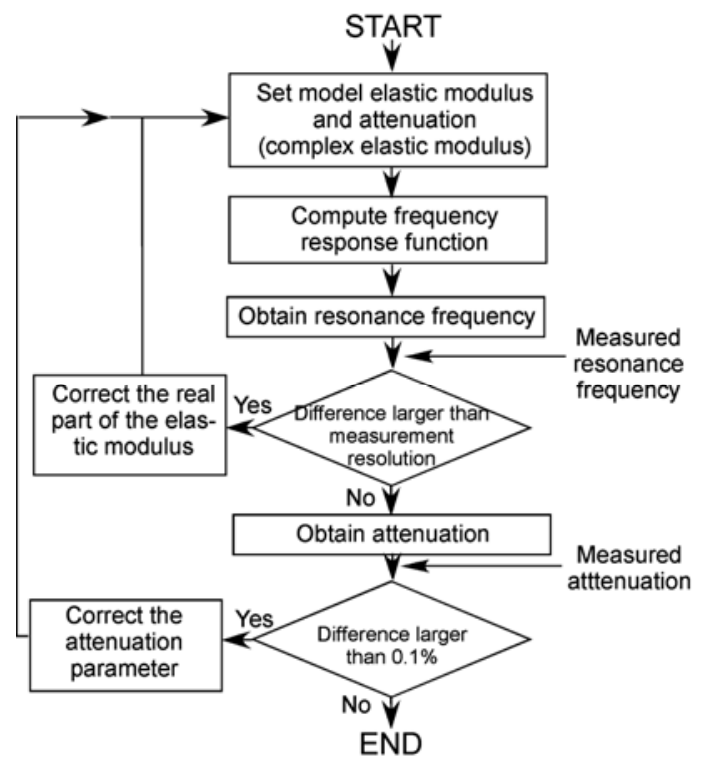

Figure 5. An example of heterogeneous stress state near a sample-bar interface resulting from uniaxial compression. For the radial stress component $\sigma_{\mathrm{rr}}$, compression is positive.

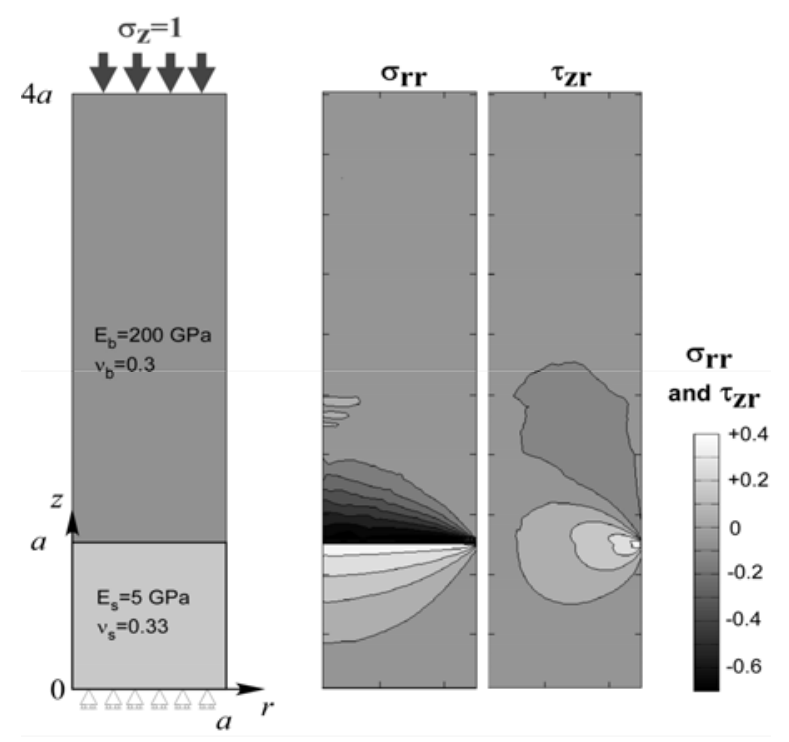


Figure 6. Idealization of stress state within a cylindrical sample for deriving effective Young's modulus of the sample considering the interface effect. section B models the constrained conical zone within the extension rods, and section I the zone within the sample. Section II models the unconstrained, uniaxially loaded part of the sample.

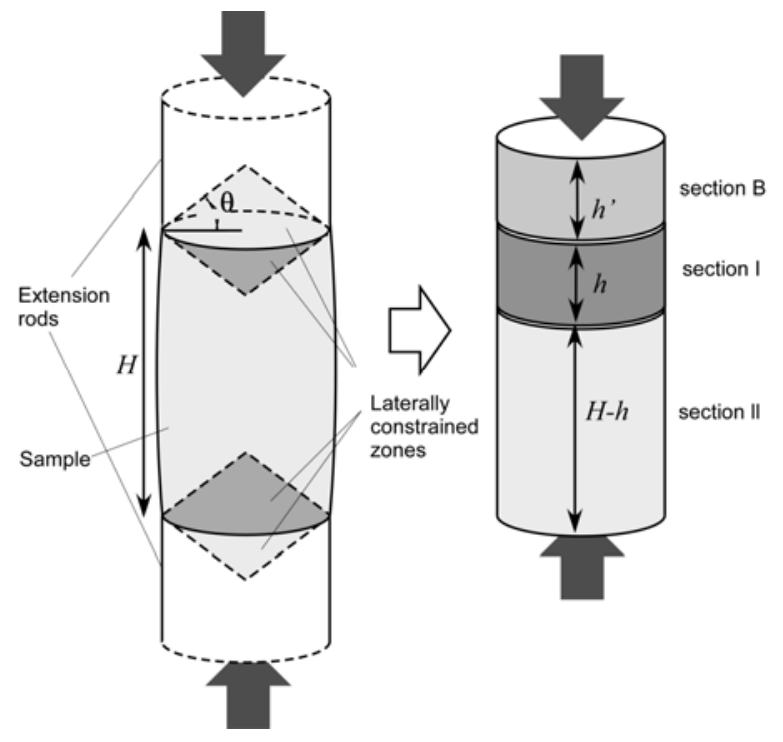

Figure 7. Comparison between analytical prediction (solid curves) and numerical results (discrete dots) for predicting the value of apparent Poisson's ratio $\bar{v}_{s}=2 \bar{E}_{s} / G-1$. Note that $D=2 a$.

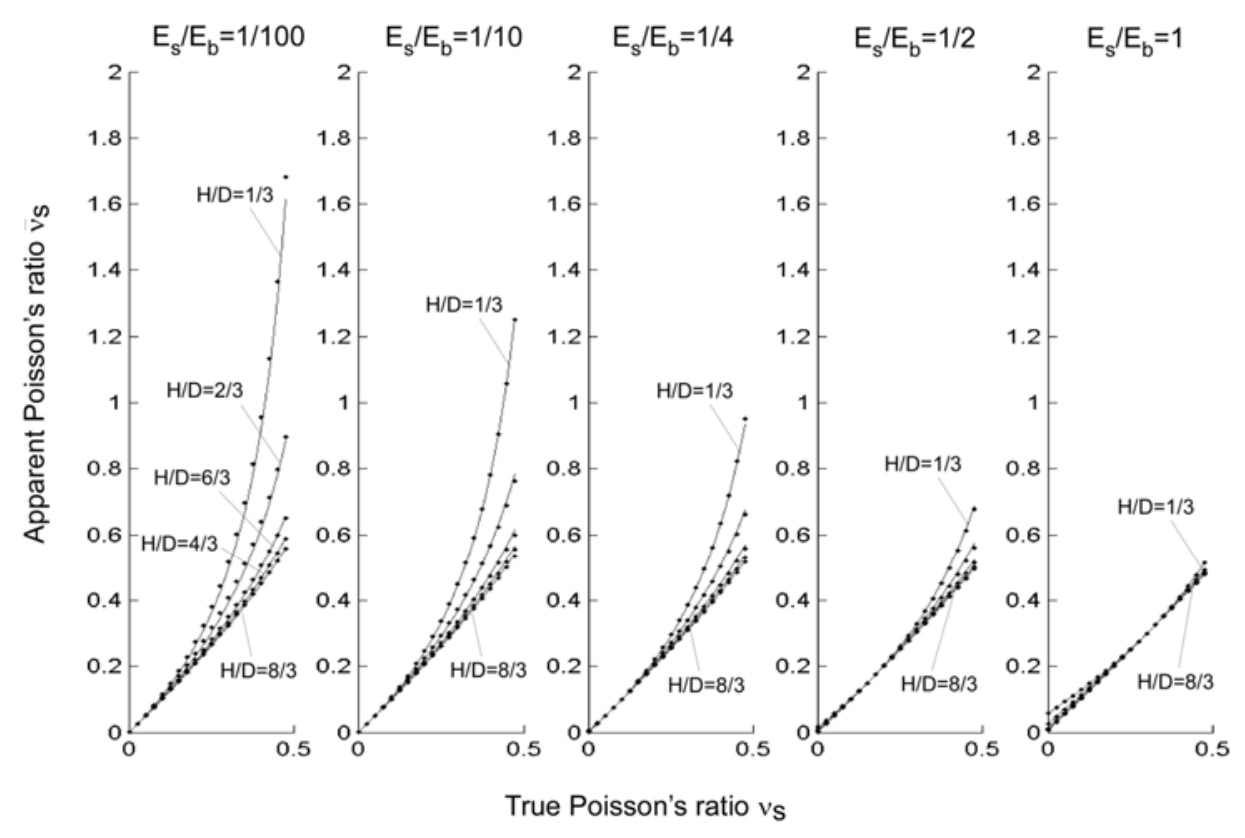


Figure 8. Measured amplitude frequency responses of acrylic samples from an SHRB test (a) and a conventional resonant bar test (b). The darker lines are for extension-mode vibrations and the lighter lines for torsion-mode vibrations.

(a) SHRB test

(b) Conventional resonant bar test

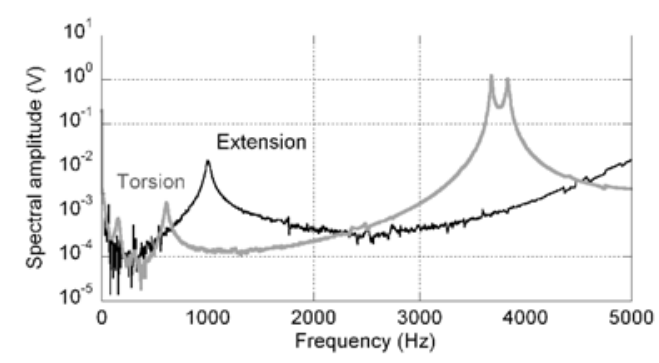

(a) SHRB tests

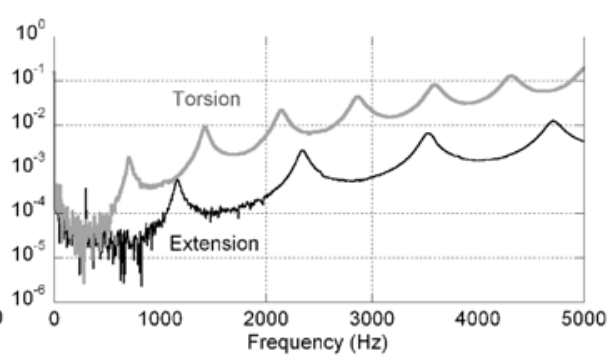

(b) Conventional resonant bar tests 
Figure 9. Elastic moduli and related attenuation parameters of polycarbonate and acrylic samples. The open symbols connected by solid lines are the results of SHRB tests. The closed, discrete symbols are from conventional resonant bar tests. The symbols connected by broken lines are the results of SHRB tests without corrections for the interface effect.

(a) Moduli of polycarbonate samples

(b) Moduli of acrylic samples

(c) Attenuation of polycarbonate samples

(d) Attenuation of acrylic samples
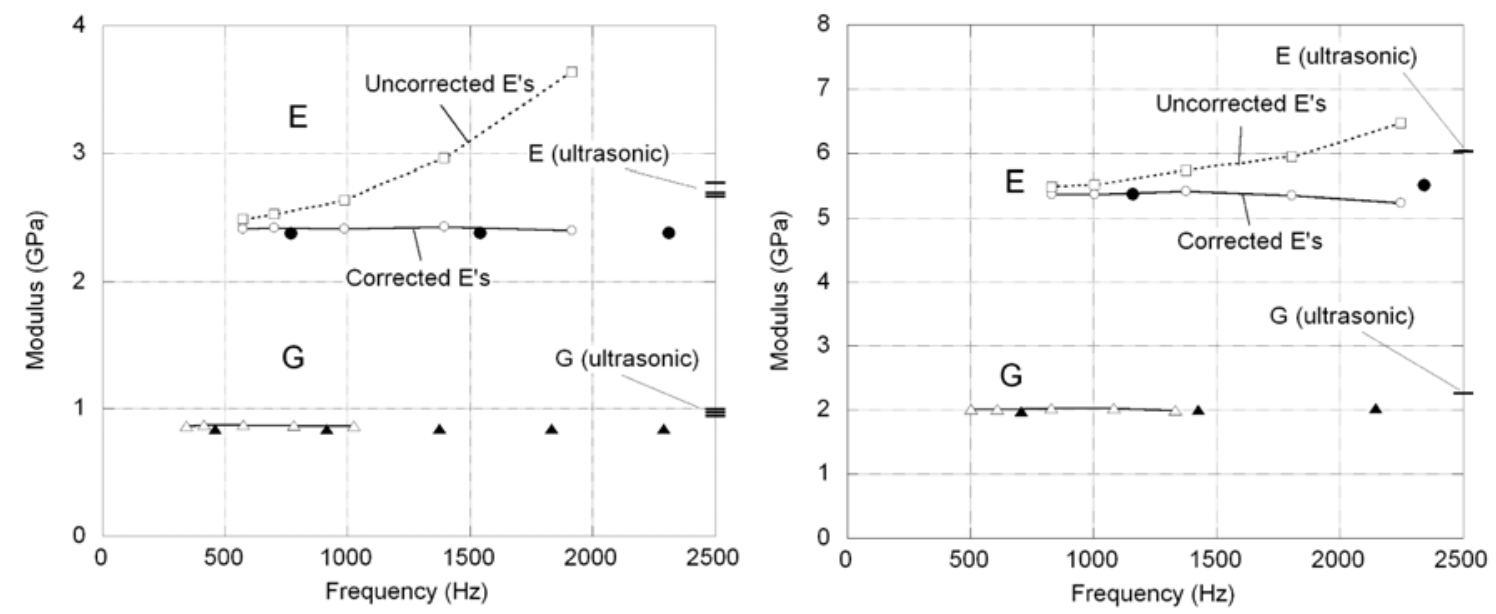

(a) Moduli of polycarbonate samples

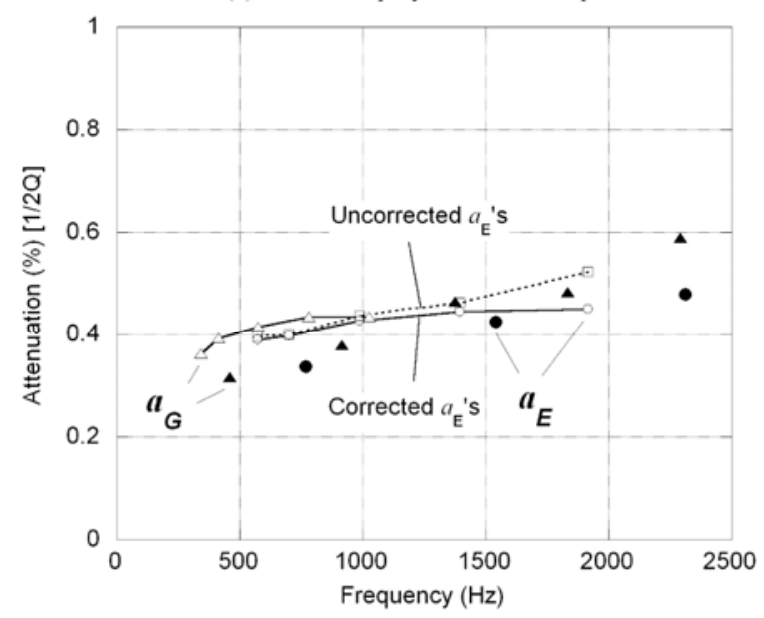

(c) Attenuation of polycarbonate samples

(b) Moduli of acrylic samples

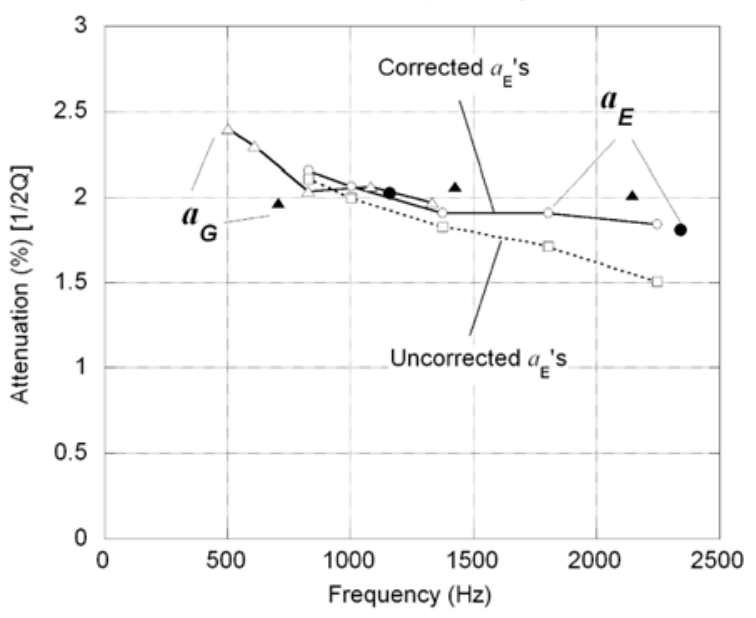

(d) Attenuation of acrylic samples 
Figure 10. P-wave velocities and attenuation determined by SHRB tests (open and closed circles) and conventional resonant bar tests (broken lines). The results of the conventional tests were obtained via curve fitting to measured moduli $E$ and $G$, and attenuation $a_{E}$ and $a_{G}$.

(a) Velocity

(b) Attenuation

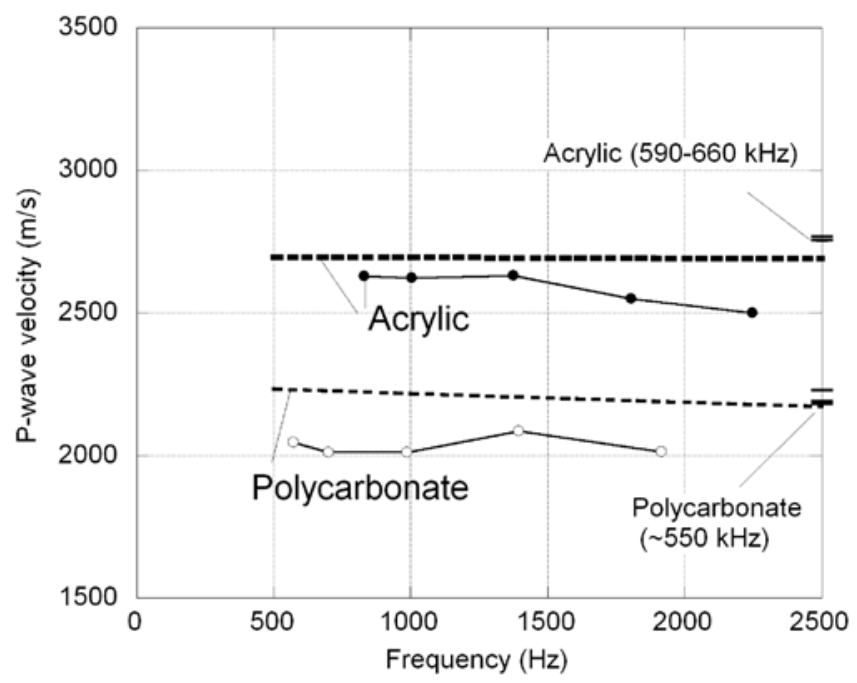

(a) Velocity

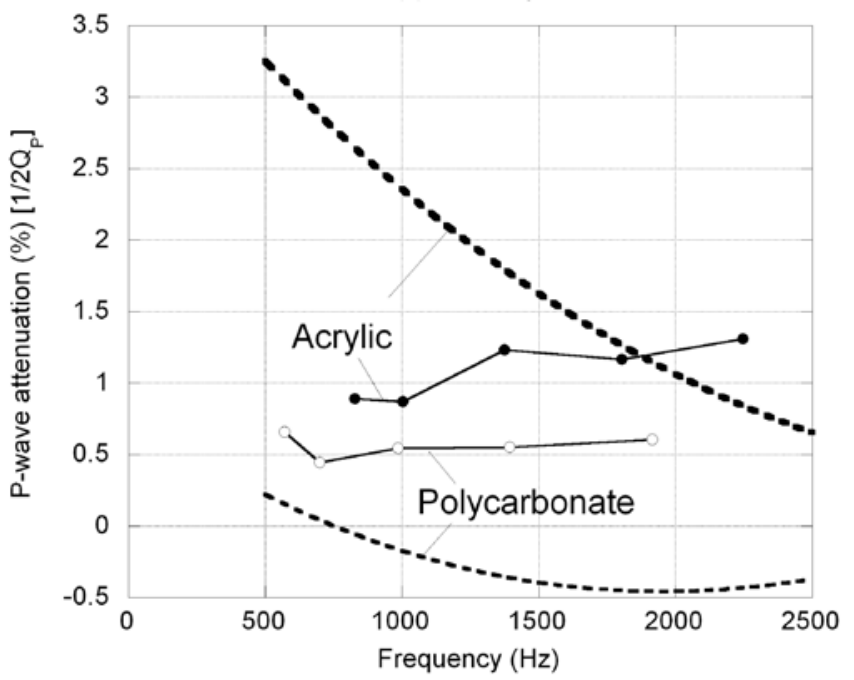

(b) Attenuation 
Figure 11. Seismic velocities and attenuation determined from resonance of a Berea sandstone core during injection into and drainage of brine from its pore space. The minimum saturation that was attained during the drainage test was approximately $55 \%$. The frequency of the test $(1 \mathrm{kHz}-$ $1.5 \mathrm{kHz}$ ) and the differences in the fluid distribution between the injection and drainage resulted in hysteresis in the seismic response.

(a) Velocities

(b) Attenuation

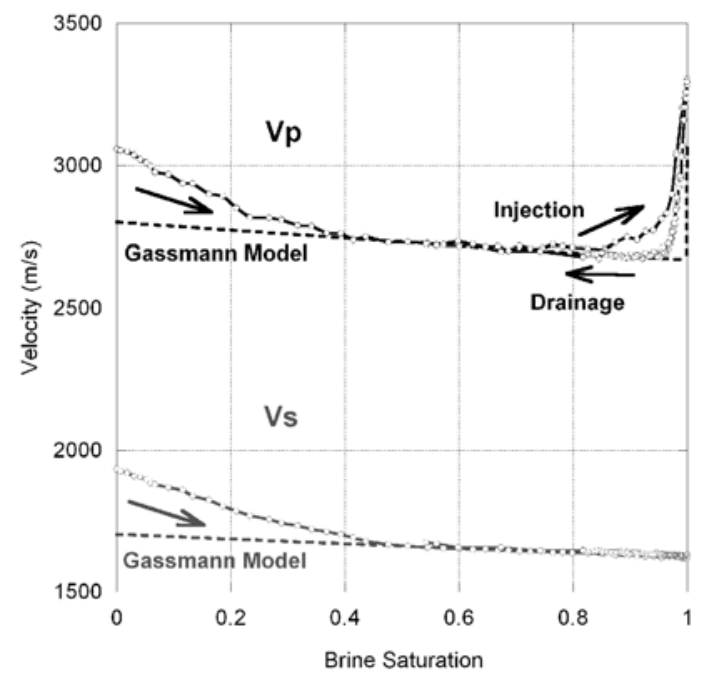

(a) Velocity

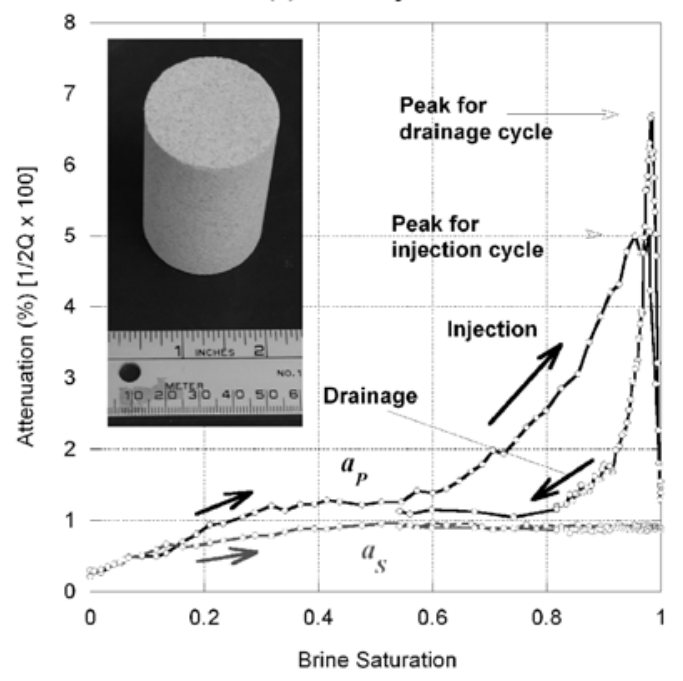

(b) Attenuation 
Figure 12. Errors in Poisson's ratio when the analytical formula in Appendix B is used. Different curves in each plot correspond to a range of H/D ratio used in Figure 7. The estimation error is contained within +/- 0.01 of Poisson's ratio value for a wide range of sample Poisson's ratio and the contrast in Young's modulus of the sample and the bar.

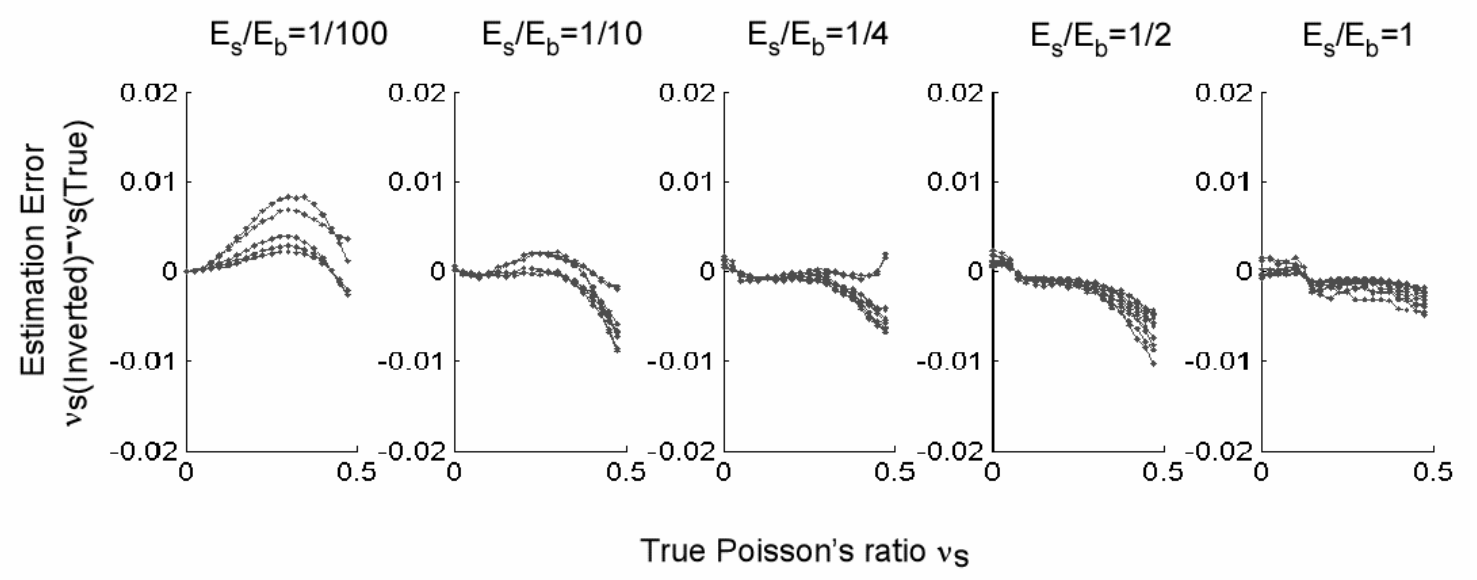




\section{DISCLAIMER}

This document was prepared as an account of work sponsored by the United States Government. While this document is believed to contain correct information, neither the United States Government nor any agency thereof, nor The Regents of the University of California, nor any of their employees, makes any warranty, express or implied, or assumes any legal responsibility for the accuracy, completeness, or usefulness of any information, apparatus, product, or process disclosed, or represents that its use would not infringe privately owned rights. Reference herein to any specific commercial product, process, or service by its trade name, trademark, manufacturer, or otherwise, does not necessarily constitute or imply its endorsement, recommendation, or favoring by the United States Government or any agency thereof, or The Regents of the University of California. The views and opinions of authors expressed herein do not necessarily state or reflect those of the United States Government or any agency thereof or The Regents of the University of California.

Ernest Orlando Lawrence Berkeley National Laboratory is an equal opportunity employer. 